\title{
Vertrauen schenken
}

\section{Daniel Schröpfer}

Dr. med., Medizinischer Direktor Stadtärztlicher Dienst Zürich, Vorstand Verein der Leitenden Spitalärzte der Schweiz (VLSS), Redaktionsmitglied

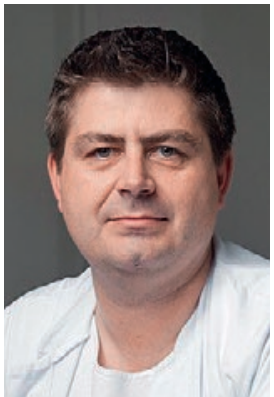

Seit mehreren Jahren bin ich in der medizinischen Grundversorgung vulnerabler Menschen tätig. Dazu gehören unter anderem die Substitutionsbehandlung, Betreuung von Asylsuchenden, Sans Papiers, Menschen ohne Krankenversicherung und weitere.

Ihre medizinische Betreuung ist - sowohl internistisch als auch psychiatrisch - sichergestellt, ihre Bedürfnisse sind gegenüber denjenigen meiner Patientinnen und Patienten meiner früheren Tätigkeit als Leiter einer internistischen Notfallstation in einem Akutspital akzentuiert. Zu Beginn meiner neuen Tätigkeit (und auch heute noch gelegentlich!) durfte ich oft die Frage beantworten: «Warum hast du gewechselt? Die Betreuung dieser Menschen ist doch so anspruchsvoll - willst du das wirklich? Ist das nicht schwierig und deprimierend?»

Die Betreuung und insbesondere Begleitung von vulnerablen Menschen stellte mich initial vor Herausforderungen. War ich es doch gewohnt, eine Lösung vorzuschlagen und auch umgehend umzusetzen. Doch was passierte? Einige Patientinnen und Patienten verliessen nach dem Gespräch unsere Räumlichkeiten, ohne mit der besprochenen Therapie begonnen zu haben. Andere haben einen Termin wahrgenommen, danach haben sie "pausiert». Wieder andere meldeten der Pflege zurück, dass der «Doktor wohl grad schwarzsieht», sie kämen später noch einmal, aktuell gehe es ihnen gut ...

Es erfordert eine besondere Aufmerksamkeit, ein geübtes Ohr, um gerade bei dieser Gruppe im Gespräch die Feinheiten ihrer Bedürfnisse und Beschwerden wahrzunehmen. Es gibt Fälle, wo ich aufgrund des Befundes und der damit verbundenen Einschränkungen eine stationäre Behandlung empfehle. Je nachdem, wie ich dies formuliere und wie die Empfehlung bei den Betroffenen ankommt, gibt es auch hier verschiedene Reaktionen beziehungsweise Verhaltensmuster: Einige konsultieren noch eine andere medizinische Fachperson aus unserem Team, andere machen ein paar Tage «Pause» und kommen in deutlich schlechterem $\mathrm{Zu}$ stand retour. Fast alle sprechen zwischendurch jedoch mit ihrer Bezugsperson in der Pflege. Dies, da sie ihre Medikation bei uns niederschwellig beziehen können und nicht immer eine Ärztin respektive einen Arzt dafür sehen müssen. Hier zeigt sich, wie wichtig es ist, im
Team zu arbeiten und zu kommunizieren. So können Mitteilungen ohne direkten Kontakt zwischen Ärztin und Patient platziert und ausgetauscht werden. Wer sich hier von der Patientin zu wenig ernst genommen fühlt oder nicht wahrgenommen, ist aus meiner Sicht fehl am Platz. Oft ist das Aushalten einer Situation, die für alle Beteiligten anspruchsvoll ist, zentral. Wir wissen, dass sich der Zustand unserer Patientinnen und Patienten schnell verändern kann - jedoch durfte ich auch wiederholt erleben, was der menschliche Körper für ein Wunderwerk der Natur ist.

Manche meiner Patienten sind seit Jahren in einer Substitutionsbehandlung. Auch bei ihnen ist die medikamentöse Therapie nicht jeden Tag gleich. Manchmal ist es von Vorteil, die Gabe des verordneten Substitutionsmedikamentes zu Beginn oder kurz vor dem ärztlichen Gespräch zu ermöglichen. Dies führt zur Stabilisierung der Patientin und in der Folge zu einer für beide Seiten fruchtbaren Konsultation. Die Annahme, die Betroffenen seien dann «zugedröhnt», ist Nonsens. Wer käme denn auf die Idee, einem «entgleisten Diabetiker» eine medikamentöse Therapie zur Normalisierung seines Blutzuckers vorzuenthalten und erst ein ausführliches Gespräch zu führen. Würde die «entgleiste Hypertonikerin» nicht initial einer entsprechenden Therapie zugeführt werden, so hätte diese wenig Verständnis oder Freude für bzw. an einer Konsultation. Die Substitutionsbehandlung ist für mich eine Dauertherapie einer chronischen Erkrankung und mit keinem Makel verbunden.

Schenken wir unseren Patientinnen und Patienten unser Vertrauen, so werden wir auch ihres erhalten. Dies ist vielleicht nicht im ersten Moment offensichtlich, doch ihr regelmässiges Erscheinen darf in diesem Sinne gewertet werden. Einige Patienten betreuen wir seit 20 Jahren. Das Ziel einer niederschwelligen Behandlung sollten aus meiner Sicht, neben der vertrauensvollen Beziehung, die Begleitung und Unterstützung der Person im Team sein - sie sagen uns, was sie brauchen.

Kurz: Ich bereue nicht einen Moment meinen Wechsel in die "Sozialmedizin" (wie man früher sagte) - es ist eine herausfordernde, Freude schenkende Arbeit, die nur in einem guten Team machbar ist. Mein Dank gilt denn auch unserem hervorragenden Team. 